Ann. Biol. anim. Bioch. Biophys., I968, 8 (4), 517-530.

\title{
INFLUENGE DE LA SUPPLÉMENTATION DES PROTÉINES DE BLÉ PAR DES DOSES CROISSANTES DE LYSINE SUR LA TENEUR EN ACIDES AMINÉS LIBRES DU SANG ET DU MUSCLE DU RAT EN CROISSANGE
}

\author{
M. PAWLAK, R. PION \\ avec la collaboration technique de Monique Allez, G. BAyle et J. Leroux \\ Laboratoire d'Etudes des Métabolismes, \\ Centre de Recherches zootechniques et vétérinaires, 63 - Theix près Clermont-Ferrand \\ Institut national de la Recherche agronomique
}

\section{SOMMAIRE}

De jeunes rats, pesant $100 \mathrm{~g}$ au début de l'expérience, reçoivent pendant deux semaines, des rations isoénergétiques et isoazotées, dont la seule source de protéine est du blé, additionné de lysine, thréonine, tryptophane et méthionine, de telle manière que tous les besoins des animaux soient couverts à l'exception du besoin en lysine. Le taux de lysine est variable et échelonné de $30 \mathrm{p}$. Ioo (blé sans lysine additionnelle) à $200 \mathrm{p}$. Ioo du besoin théorique. Des gains de poids moyens de 5,8 à 7,25 g par jour sont obtenus dans tous les cas où les besoins en lysine des animaux sont satisfaits. La teneur en lysine libre du sang augmente avec la teneur en lysine de la ration, et l'accroissement est particulièrement net lorsque le taux de lysine alimentaire dépasse le besoin.

Une augmentation similaire, mais beaucoup plus marquée, est observée dans le cas du muscle: la teneur en lysine libre des muscles des rats qui ont consommé la ration la plus riche en lysine est égale à 27 fois celle des muscles des animaux ayant consommé le blé non supplémenté en lysine, alors que les valeurs les plus élevées observées dans le sang ne sont que 7 fois supérieures aux plus faibles.

\section{INTRODUCTION}

Les acides aminés libres du sang constituent la principale forme de transport des produits de la digestion des matières azotées alimentaires, et leurs teneurs traduisent l'équilibre entre les apports et les besoins. Plusieurs auteurs, et en particulier LoNGeNECker et Hause (I959) chez le Chien, Morrisson, Middleton et MCLaughlan (I96I) chez le Rat, Zimmerman et Scotr (r965) chez le Poulet, ont mis en évidence une 
relation entre la composition en acides aminés libres du plasma sanguin et la composition des matières azotées ingérées.

Or, il y a également un équilibre entre apport et utilisation au niveau des tissus consommateurs d'acides aminés, et en particulier du muscle, qui constitue la masse azotée la plus importante de l'organisme, et est le siège, en période de croissance, d'une synthèse protéique intense. De plus, HARPER et RogERs (1965) ont constaté chez le Rat que des déséquilibres en acides aminés modifiaient plus les teneurs en acides aminés libres du muscle que celles du sang.

Aussi avons-nous entrepris l'étude comparative de l'influence de la composition des protéines alimentaires sur la composition en acides aminés libres du sang et du muscle chez le Rat en croissance. Après avoir constaté, dans une première expérience, (PAWLAK et Pron, 1967) qu'une forte carence en lysine modifiait beaucoup plus la teneur en lysine du muscle que celle du sang, nous avons cherché à déterminer dans la présente expérience l'influence de doses croissantes de lysine, allant d'une forte carence à un excès, sưr la teneur en acides aminés libres de ces tissus.

\section{MATÉRIEL ET MÉTHODES}

\section{Animaux}

Des rats mâles de souche $W$ istar, élevés dans des locaux éclairés de $6 \mathrm{~h}$ à $\mathrm{I} 8 \mathrm{~h}$, maintenus à $22^{\circ}$ et de degré hygrométrique contrôlé (6o p. I00), reçoivent un régime standard depuis le sevrage jusqu'à ce qu'ils atteignent un poids de $100 \mathrm{à} \mathrm{I} I 0 \mathrm{~g}$ soit pendant $\mathrm{I} 0$ à $\mathrm{I} 2$ jours. Ils sont alors répartis en lots de 8 dont les poids moyens ne diffèrent pas les uns des autres de plus de $2 \mathrm{~g}$.

Ils sont élevés en cages individuelles et reçoivent à volonté les rations expérimentales sous forme semi-liquide pendant 2 semaines. Les consommations sont mesurées chaque jour, et la croissance est contrôlée par pesée tous les 2 jours. Les différents lots ont été répartis en quatre expériences (tabl .5) effectuées à des époques différentes.

\section{Régimes}

Ils sont à base de blé de deux variétés différentes, ayant subi une conservation de durée variable après la récolte (tabl. I), additionné de minéraux et de vitamines (tabl. 2 et 3 ) et supplémenté en DLméthionine, L-tryptophane et L-thréonine (tabl. 4), ainsi que par des doses de L-lysine variables selon les lots (tabl. 5). Le taux protéique de la ration est voisin de $x 2 \mathrm{p}$. 1oo; l'équilibre énergétique (4 500 kilocalories par $\mathrm{kg}$ de matière sèche) est assuré par addition d'amidon de maîs et d'huile d'arachide en modifiant les proportions d'amidon de maïs et d'huile d'arachide.

TABLEAU I

Caractéristiques des blés utilisés

\begin{tabular}{|c|c|c|c|c|}
\hline Expérience & Variété & $\begin{array}{c}\text { Durée } \\
\text { de conservation }\end{array}$ & $\begin{array}{c}\text { Matière sèche } \\
(\%)\end{array}$ & $\begin{array}{c}\text { Matière azotée } \\
(\mathbf{N} \times 6,25) \\
(\% \text { de MS })\end{array}$ \\
\hline I & Étoile de Choisy & 12 mois & 87,5 & 14,7 \\
\hline II & Aronde & 3 mois & 87,5 & 14,1 \\
\hline III & 一 & 6 mois & 87,5 & 14,1 \\
\hline IV & - & $12 \mathrm{mois}$ & 87,5 & 14,1 \\
\hline
\end{tabular}


TABLEAUX 2, 3, 4

Apport minéral par $\mathrm{kg}$ de $M S$ ingérée

\begin{tabular}{|c|c|c|c|}
\hline $\mathrm{Ca}$ & $10 \mathrm{~g}$ & $\mathrm{Mn}$ & $30 \mathrm{mg}$ \\
\hline $\mathrm{P}$ & $3 \mathrm{~g}$ & $\mathrm{Zn}$ & $10 \mathrm{mg}$ \\
\hline $\mathrm{Na}$ & $2 \mathrm{~g}$ & F & $5 \mathrm{mg}$ \\
\hline $\mathrm{Fe}$ & $30 \mathrm{mg}$ & I & $150 \mu \mathrm{g}$ \\
\hline $\mathrm{Cu}$ & $5 \mathrm{mg}$ & Co & $150 \mu \mathrm{g}$ \\
\hline
\end{tabular}

Complément vitaminique par kg de MS ingérée

\begin{tabular}{|c|c|c|}
\hline $\mathrm{B}_{12}$ (cyanocobalamine) & 0,05 & $\mathrm{mg}$ \\
\hline Acide folique $\ldots \ldots \ldots \ldots \ldots \ldots \ldots$ & 1 , & — \\
\hline Vitamine $\mathrm{E} \ldots \ldots \ldots \ldots \ldots \ldots \ldots$ & 100 & - \\
\hline Vitamine $\mathrm{K} \ldots \ldots \ldots \ldots \ldots \ldots \ldots$ & 2 & - \\
\hline Thiamine $\mathrm{HCl} \ldots \ldots \ldots \ldots \ldots \ldots \ldots$ & 10 & - \\
\hline Acide nicotinique $\ldots \ldots \ldots \ldots \ldots \ldots \ldots$ & 30 & - \\
\hline Pyridoxine $\mathrm{HCl} \quad \ldots \ldots \ldots \ldots \ldots \ldots \ldots$ & 10 & 一 \\
\hline Riboflavine $\ldots \ldots \ldots \ldots \ldots \ldots \ldots \ldots$ & 15 & - \\
\hline Pantothénate de calcium $\ldots \ldots \ldots \ldots \ldots$ & 50 & - \\
\hline Inositol $\ldots \ldots \ldots \ldots \ldots \ldots \ldots \ldots$ & 300 & - \\
\hline Acide para-aminobenzoiqque $\ldots \ldots \ldots \ldots \ldots$ & 100 & - \\
\hline Acide ascorbique $\ldots \ldots \ldots \ldots \ldots \ldots$ & 100 & - \\
\hline 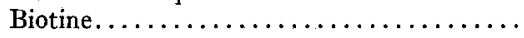 & 0,2 & - \\
\hline Choline chlorure $\ldots \ldots \ldots \ldots \ldots \ldots \ldots \ldots$ & 1000 & - \\
\hline Vitamine $A \ldots \ldots \ldots \ldots \ldots \ldots \ldots$ & 4000 & UI \\
\hline Vitamine $\mathrm{D}_{2}, \ldots \ldots \ldots \ldots \ldots \ldots \ldots$ & 2000 & UI \\
\hline
\end{tabular}

Acides aminés en $\mathrm{g}$ par $\mathrm{kg}$ de $M S$ ingérée

\begin{tabular}{|c|c|c|c|}
\hline & Provenant du blé (i) & Supplémentaire & total \\
\hline 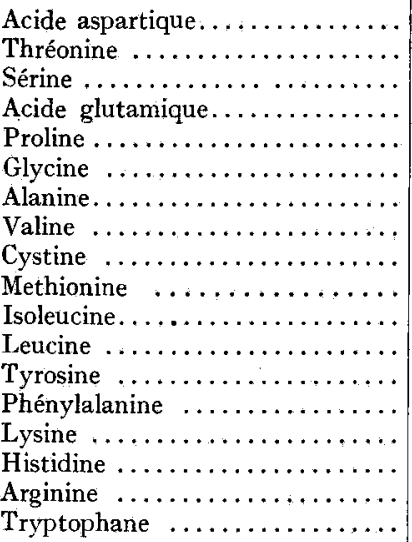 & $\begin{array}{c}5,55 \\
3,1 \\
5,45 \\
38,8 \\
11,35 \\
4,6 \\
4,2 \\
5,6 \\
2,9 \\
1,75 \\
4,4 \\
7,9 \\
3,6 \\
5,4 \\
2,9 \\
2, \mathbf{C 5} \\
\mathbf{5 , 4 5}\end{array}$ & $\begin{array}{c}1,2 \\
\text { Cf. tableau } \\
0,6\end{array}$ & $\begin{array}{c}5,55 \\
5,4 \\
5,45 \\
38,8 \\
11,35 \\
4,6 \\
4,2 \\
5,6 \\
2,9 \\
2,95 \\
4,4 \\
7,9 \\
3,6 \\
5,4 \\
2,9 \\
2,65 \\
5,45\end{array}$ \\
\hline
\end{tabular}

(1) Pion et Fauconneau (1966). 
M. PAWLAK, R. PION

\begin{tabular}{|c|c|c|c|c|c|c|}
\hline$\geq$ & $\stackrel{\oplus}{*}$ & $\stackrel{\infty}{\rightarrow}$ & $\stackrel{\infty}{\sim}$ & : & 8 & $\begin{array}{l}m_{2} \\
=\end{array}$ \\
\hline$\Xi$ & के & 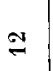 & \pm & $\begin{array}{l}0 \\
\infty \\
\infty\end{array}$ & $\underset{ }{\approx}$ & $\begin{array}{l}x^{2} \\
\stackrel{\sim}{\sim}\end{array}$ \\
\hline$\Xi$ & $\mathscr{s}$ & $\exists$ & \pm & $\stackrel{\vec{x}}{\stackrel{*}{\infty}}$ & $n$ & $\stackrel{\Rightarrow}{\Rightarrow}$ \\
\hline$\Xi$ & $\stackrel{\mathscr{I}}{\circ}$ & 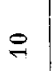 & \pm & $\stackrel{\infty}{=}$ & $\therefore$ & $\stackrel{8}{\circ}$ \\
\hline$\Xi$ & 哭 & $\infty$ & $\stackrel{H}{=}$ & $\stackrel{N}{5}$ & 8 & s. \\
\hline$\gtrsim$ & $\Xi$ & $r$ & $\stackrel{\infty}{\sim}$ & $\begin{array}{c}\text { and } \\
\text { is }\end{array}$ & 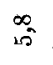 & $\begin{array}{l}12 \\
\infty^{2}\end{array}$ \\
\hline$\Xi$ & $\underset{\sim}{\infty}$ & $n$ & $\stackrel{*}{*}$ & $\begin{array}{l}0 \\
\stackrel{\infty}{\sim}\end{array}$ & $\vec{\sigma}$ & $\infty$ \\
\hline$w$ & $\approx$ & 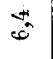 & $\stackrel{9}{\sim}$ & $\begin{array}{l}\stackrel{g}{0} \\
\stackrel{0}{0}\end{array}$ & is & $\infty$ \\
\hline 寻 & న & 0 & \pm & 5 & 5 & $=$ \\
\hline- & 27 & \begin{tabular}{l}
$\infty$ \\
\multirow{4}{*}{}
\end{tabular} & $\stackrel{\infty}{\sim}$ & $\stackrel{0}{\stackrel{0}{\approx}}$ & $\stackrel{\infty}{i \infty}$ & : \\
\hline-1 & $\stackrel{\Im}{\sim}$ & $\stackrel{\infty}{\infty}$ & $\stackrel{2}{\sim}$ & $\stackrel{+\infty}{\sim}$ & $\hat{\text { is }}$ & $\begin{array}{l}28 \\
30 \\
20\end{array}$ \\
\hline$\Xi$ & $\ddot{~}$ & $\Leftrightarrow$ & 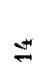 & $\stackrel{g}{\leftrightarrow}$ & 8 & $\stackrel{10}{10}$ \\
\hline- & $\Rightarrow$ & $\stackrel{\leftrightarrow}{\rightarrow}$ & $\stackrel{\Re}{\sim}$ & $\stackrel{n}{\infty}$ & $\xi$ & $\xi$ \\
\hline$\Xi$ & $\Omega_{i}$ & 0 & $\stackrel{ \pm}{*}$ & $\ddot{0}$ & $\overbrace{\infty}^{\infty}$ & is \\
\hline 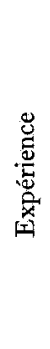 & & 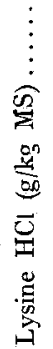 & 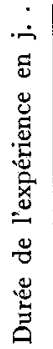 & 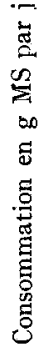 & 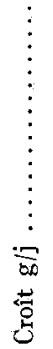 & 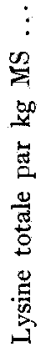 \\
\hline
\end{tabular}




\section{Prélèvement des échantillons}

En fin de période expérimentale, les rats sont abattus le matin entre to et $12 \mathrm{~h}$ sans avoir subi de jeûne préalable.

Le sang carotide est prélevé sous anesthésie à l'éther et recueilli directement dans sept fois son volume d'éthanol froid $\left(-10^{\circ}\right)$.

Les muscles des membres postérieurs sont disséqués et placés immédiatement dans l'azote liquide. Les prélèvements correspondant à l'ensemble des animaux de chaque lot sont regroupés pour l'analyse. Les échantillons sont conservés à-20 $20^{\circ} \mathrm{C}$.

\section{Extractions}

a) Acides aminés libres des sangs.

L'agent d'extraction est l'éthanol 84 p. Ioo utilisé à froid. Une quantité connue de norleucine est ajoutée à l'échantillon afin de contrôler le caractère quantitatif des différentes opérations. La mise en présence des composés à extraire et du solvant est effectuée par broyage (quatre fois I $\mathrm{mn}$ ) au Waring Blendor. Les pots sont refroidis entre deux broyages successifs.

Les extraits sont séparés du résidu par centrifugation à froid $(4500 \mathrm{~g})$. Le résidu est repris et extrait à nouveau, et l'ensemble des manipulations est répété quatre fois. Les différents extraits (700 à $800 \mathrm{ml}$ pour 20 à $30 \mathrm{~g}$ de sang frais) obtenus sont regroupés, amenés à un $\mathrm{pH}$ compris entre 3 et 4 par addition d'acide acétique glacial et purifiés par passage sur échangeurs de cations (Amberlite IR I 20) sous forme $\mathrm{H}^{+}$qui fixe les acides aminés et amides et les cations minéraux.

Les composés non retenus sont éliminés par lavage à l'eau bidistillée, puis la majorité des acides aminés et amides sont élués par une solution d'ammoniaque $2 \mathrm{~N}(\mathrm{I}, 5$ fois le volume de la résine) suivie d'un rinçage par un égal volume d'eau bidistillée. Les plus fortement retenus sont élués par une solution d'ammoniaque $4 \mathrm{~N}$ ( 3 fois le volume de la résine) suivie d'un rinçage à l'eau bidistillée.

Les extraits sont mélangés et l'ammoniac est éliminé par évaporation sous vide (source chaude $3^{\circ}$, source froide $-20^{\circ}$ ). Ils sont alors concentrés et repris par un volume connu de tampon citrate $\mathrm{pH}_{2,2}$ en vue du dosage des acides aminés par chromatographie sur colonne.

\section{b) Acides aminés libres des muscles.}

Les échantillons additionnés de norleucine sont extraits par l'acide picrique à I p. Ioo (TALlan, MOORE et STEIN, I954), selon une technique d'épuisements successifs similaire à celle employée pour l'extraction des acides aminés libres des sangs. Le volume total des extraits est de 600 à $700 \mathrm{ml}$ pour 40 à $5 \circ \mathrm{g}$ de muscle frais.

L'acide picrique est éliminé par passage des extraits sur une colonne de résine échangeuse d'anions Dowex $2 \times 8$ (50 à roo mesh). Les acides aminés ne sont pas retenus sur cette résine et sont recueillis dans l'effluent, qui est concentré par évaporation sous vide. L'échantillon est ensuite trai té (MOORE et STEIN, I958), de manière à éliminer l'interférence du glutathion dans le fractionnement des acides aminés, puis ajusté à un volume connu à l'aide de tampon citrate $\mathrm{pH} \mathbf{2 , 2}$ en vue du dosage.

\section{Séparation et dosage des acides aminés.}

Ils sont séparés et dosés sur appareil automatique (Spackman, Moore et STEIN, I958). Les acides aminés acides et neutres sont séparés en $15 \mathrm{~h}$ sur une colonne de $140 \times 0,9 \mathrm{~cm}$ de résine AMINEX $Q$ I $5^{\circ} \mathrm{S}$ (Bio Rad Laboratories) maintenue à $50^{\circ} \mathrm{C}$, au moyen de tampons citrates de sodium $\mathrm{pH} 3,25$ et 4,25 (MoORE, SPackman et StEIN, 1958). Le débit utilisé est de $45 \mathrm{ml}$ par heure, et le changement de tampon est effectué $7 \mathrm{~h}$ i5 $\mathrm{mn}$ après le début de l'élution.

Les acides aminés basiques, l'ammoniac, l'ansérine et la carnosine sont séparés en $7 \mathrm{~h}$ sur une colonne de $25 \times 0,9 \mathrm{~cm}$ de résine XX $93730 \circ$ (Phoenix Précision Instrument).

L'élution est effectuée d'abord à $40^{\circ}$ avec du tampon citrate $0,35 \mathrm{~N} \mathrm{pH} 4,26$ jusqu'à la sortie de l'ansérine, puis à $5^{\circ}$ avec du tampon citrate $0,35 \mathrm{~N} \mathrm{pH} 5,28$. Le débit du tampon est de $5 \circ \mathrm{ml}$ par heure.

Le mode de fractionnement utilisé pour les acides aminés acides et neutres ne permet pas de séparer la sérine de la glutamine, et la proline de la citrulline.

\section{RÉSULTATS}

Les différents essais de supplémentation des régimes à base de blé par la lysine ont été réalisés en quatre expériences, dont nous analyserons séparément les résultats. 


\section{Croissance et consommation (tab1. 5)}

Expérience $I$. Les taux de lysine utilisés sont tous inférieurs aux besoins de l'animal. L'importante différence de croissance (plus de $35 \mathrm{p}$. Ioo) observée entre les lots II et I2 est obtenue sans augmentation importante de la consommation d'aliment. L.es doses de lysine supplémentaires dans les régimes consommés par les animaux des lots I3 et I4 n'entraînent qu'une très faible augmentation de croissance. En revanche, les consommations alimentaires sont légèrement diminuées (respectivement de 4 à 8 p. IOo), et l'indice de consommation est amélioré.

\section{TABLEAU 6}

Expérience I

Teneurs en acides aminés libres

(en $\mathrm{mg}$ pour $100 \mathrm{~g}$ de tissu frais)

\begin{tabular}{|c|c|c|c|c|c|c|c|c|}
\hline \multirow[b]{2}{*}{ Lot $n^{0}$} & \multicolumn{4}{|c|}{ Sangs } & \multicolumn{4}{|c|}{ Muscles } \\
\hline & 11 & 12 & 13 & 14 & 11 & 12 & 13 & 14 \\
\hline Lysine ajoutée ( $\mathrm{g} / \mathrm{kg}$ d'aliment $\mathrm{sec})$.. & 1,3 & 2,6 & 3,8 & 5,1 & 1,3 & 2,6 & 3,8 & 5,1 \\
\hline Croît en $\mathrm{g} /$ jour $\ldots \ldots \ldots \ldots \ldots \ldots \ldots$ & 4,2 & 5,7 & 5,8 & 5,9 & 4,2 & 5,7 & 5,8 & 5,9 \\
\hline Acide aspartique $\ldots \ldots \ldots \ldots \ldots \ldots$ & 0,4 & 0,3 & 0,3 & 0,3 & 4,3 & 4,6 & 5,4 & 5,8 \\
\hline Thréonine ...... & 4,5 & 3,7 & 4,0 & 3,8 & 19,9 & 16,6 & 16,9 & 19,5 \\
\hline Sérine + glutamine & 12,1 & 9,7 & - & - & 80,9 & 55,0 & 71,8 & - \\
\hline Acide glutamique $\ldots \ldots \ldots \ldots \ldots \ldots$ & 2,9 & 3,9 & - & - & 40,7 & 48,6 & - & - \\
\hline Glycine $\ldots \ldots \ldots, \ldots, \ldots, \ldots$ & 2,3 & 2,3 & 2,8 & 2,4 & 36,9 & 33,7 & 40,7 & 38,8 \\
\hline Alanine $\ldots \ldots \ldots \ldots \ldots \ldots \ldots \ldots$ & 4,0 & 3,4 & 3,7 & 3,4 & 33,5 & 29,0 & 35,0 & 34,1 \\
\hline Valine $\ldots \ldots \ldots \ldots \ldots \ldots \ldots \ldots \ldots$ & 1,1 & 1,0 & 1,0 & 1,0 & 2,9 & 2,3 & 2,4 & 2,6 \\
\hline Méthionine $\ldots \ldots \ldots \ldots \ldots \ldots \ldots \ldots$ & 0,5 & 0,4 & 0,5 & 0,4 & - & - & - & - \\
\hline Isoleucine $\ldots \ldots \ldots \ldots \ldots \ldots \ldots \ldots$ & 0,6 & 0,4 & 0,5 & 0,6 & 2,2 & 1,5 & 1,8 & 2,2 \\
\hline Leucine $\ldots \ldots \ldots \ldots \ldots \ldots \ldots \ldots$ & 1,0 & 0,7 & 1,0 & 1,2 & 4,1 & 3,3 & 3,4 & 7,6 \\
\hline Tyrosine ....... & 0,8 & 0,7 & 0,9 & 0,9 & 3,9 & 2,8 & 2,8 & 3,4 \\
\hline Phénylalanine ... & 0,8 & 0,7 & 0,8 & 0,9 & 3,6 & 2,7 & 2,3 & 3,1 \\
\hline Ornithine .... & 0,7 & 0,6 & 0,6 & 0,7 & 1,1 & 0,8 & 0,8 & - \\
\hline Lysine ... & 1,3 & 2,3 & 2,7 & 3,8 & 2,7 & 3,6 & 4,1 & 8,5 \\
\hline Histidine & 0,8 & 0,6 & 0,7 & 0,7 & 3,8 & 4,3 & 3,8 & 3,5 \\
\hline Arginine $\ldots \ldots \ldots \ldots$ & 2,4 & 2,9 & 1,3 & 3,5 & 5,1 & 3,9 & 3,6 & 4,9 \\
\hline
\end{tabular}

Expérience $I I$. - Les croissances obtenues sont, à taux de lysine égal, un peu supérieures à celles de l'expérience précédente. Les taux de supplémentation les plus élevés, choisis 1'un pour satisfaire largement les besoins du Rat, et l'autre pour lui apporter un net excès, entraînent une augmentation de croissance, au prix d'un léger accroissement de la consommation.

L'amélioration importante de la croissance (qui est presque doublée) permise par l'addition au blé de $2, \mathrm{I} 6 \mathrm{~g}$ de lysine est obtenue avec une augmentation relativement faible de la consommation (de l'ordre de 4,5 p. Ioo).

Expérience $I I I$. - Dans cette expérience, les deux taux de lysine alimentaire les plus faibles correspondent sensiblement à la satisfaction du besoin, tandis que les deux taux supérieurs correspondent à un excès : les croissances obtenues sont toutes élevées. La croissance tend à augmenter plus vite que la consommation. 
Expérience $I V$. - Un excès de lysine alimentaire (environ deux fois le besoin) a été comparé à un régime théoriquement équilibré. Les croissances obtenues dans les deux cas sont voisines de celles qui avaient été observées dans l'expérience I.

\section{Composition en acides aminés libres $d u$ sang et du muscle (tabl. 6 à 9)}

Certains composés azotés, qui ne figurent pas sur les tableaux de résultats, sont présents dans les tissus analysés. C'est le cas notamment de la taurine, de la carnosine et de 1'ansérine, que 1'on trouve dans les muscles en quantités beaucoup plus importantes que les acides aminés. La proline, la citrulline, et, en faible proportion, la cystine sont présentes dans les deux tissus, mais n'ont pas été dosées quantitativement.

\section{TABLEAU 7}

Expérience II

Teneurs en acides aminés libres (en mg pour roo $\mathrm{g}$ de tissu frais)

\begin{tabular}{|c|c|c|c|c|c|c|c|c|}
\hline \multirow{3}{*}{$\begin{array}{l}\text { Lots } n^{0} \\
\text { Lysine ajoutée }(g / k g \text { d'aliment sec).. }\end{array}$} & \multicolumn{4}{|c|}{ Sangs } & \multicolumn{4}{|c|}{ Muscles } \\
\hline & 23 & 24 & 25 & 26 & 23 & 24 & 25 & 26 \\
\hline & 0 & 2,6 & 6,4 & 8,0 & 0 & 2,6 & 6,4 & 8,0 \\
\hline Croît en $\mathrm{g} /$ jour ... & 3,3 & 6,0 & 6,5 & 7,0 & 3,3 & 6,0 & 6,5 & 7,0 \\
\hline Acide aspartique & 0,7 & 0,7 & 1,0 & - & 5,8 & 5,2 & 5,5 & 7,7 \\
\hline Thréonine ..... & 8,8 & 8,2 & 6,0 & 6,2 & 29,8 & 24,3 & 23,0 & 18,4 \\
\hline Sérine + glutamine & 14,1 & 13,3 & 15,9 & 12,3 & 83,1 & 79,0 & 70,7 & 66,3 \\
\hline Acide glutamique...$\ldots \ldots$ & 2,7 & 3,7 & 3,8 & 3,8 & 43,7 & 45,6 & 42,4 & 47,0 \\
\hline Glycine..$\ldots \ldots \ldots \ldots \ldots \ldots \ldots \ldots$ & 2,3 & 2,7 & 2,7 & 2,5 & 33,5 & 37,9 & 35,0 & 35,6 \\
\hline Alanine .... & 3,5 & 3,9 & 3,8 & 3,8 & 24,3 & 26,5 & 27,7 & 27,8 \\
\hline Valine .......... & 1,7 & 2,0 & 2,0 & 1,9 & 1,6 & 2,1 & 1,8 & 2,3 \\
\hline Méthionine ....... & 0,4 & 0,8 & 0,8 & 1,0 & $\mathbf{0 , 6}$ & 0,8 & 0,9 & 1,1 \\
\hline Isoleucine $\ldots \ldots \ldots$ & 0,8 & 1,0 & 1,1 & 1,1 & 0,8 & 1,2 & 1,1 & 1,4 \\
\hline Leucine $\quad . . . .$. & 1,3 & 1,5 & 1,9 & 1,8 & 1,2 & 1,6 & 1,7 & 2,0 \\
\hline Tyrosine .......... & 0,8 & 1,1 & 1,1 & 1,1 & 1,3 & 1,6 & 1,6 & 1,8 \\
\hline Phénylalanine...$\ldots \ldots \ldots \ldots \ldots \ldots \ldots \ldots$ & 0,8 & 1,0 & 1,2 & 1,2 & 0,8 & 1,0 & 1,0 & 1,3 \\
\hline Ornithine...$\ldots \ldots \ldots \ldots \ldots \ldots \ldots$ & 0,7 & 0,5 & 0,6 & 0,5 & 1,5 & 1,0 & 1,0 & 1,1 \\
\hline Lysine $\ldots \ldots \ldots \ldots \ldots \ldots \ldots \ldots \ldots \ldots \ldots$ & 1,3 & 2,4 & 5,9 & 6,4 & 1,8 & 4,6 & 22,6 & 22,9 \\
\hline Histidine...$\ldots$. & 0,9 & 0,9 & 1,0 & 1,1 & 2,5 & 3,1 & 1,5 & 3,7 \\
\hline Arginine...$\ldots \ldots \ldots \ldots \ldots$ & 1,7 & 2,0 & 2,2 & 3,1 & 4,0 & 3,1 & 5,0 & 5,4 \\
\hline
\end{tabular}

\section{Composition comparée des tissus sanguins et musculaires.}

I a teneur en acides aminés libres et amides libres totaux des muscles des animaux recevant un régime équilibré est environ dix fois plus élevée que celle des sangs correspondants. Cette différence est due, en majeure partie, à la présence dans le muscle de quantités importantes de glycine, ainsi que d'acide aspartique, d'acide glutamique et d'alanine, de glutamine et de sérine. Les différences observées dans le cas des acides aminés indispensables sont beaucoup plus faibles, et ne concernent que certains d'entre eux : la thréonine, 1'histidine, la lysine et, à un moindre degré, l'arginine. 


\section{TABLEAU 8}

\section{Expérience III}

Teneurs en acides aminés libres

(en $\mathrm{mg}$ pour Ioo $\mathrm{g}$ de tissu frais)

\begin{tabular}{|c|c|c|c|c|c|c|c|c|}
\hline \multirow{3}{*}{$\begin{array}{l}\text { Lot } n^{\circ} \ldots \ldots \ldots \ldots \ldots \ldots \ldots \ldots \ldots \ldots \\
\text { Lysine ajoutée }(g / k g \text { d'aliment sec) .. }\end{array}$} & \multicolumn{4}{|c|}{ Sangs } & \multicolumn{4}{|c|}{ Muscles } \\
\hline & 27 & 28 & 29 & 30 & 27 & 28 & 29 & 30 \\
\hline & 4,8 & 5,6 & 8,8 & 9,6 & 4,8 & 5,6 & 8,8 & 9,6 \\
\hline Crô̂t en $\mathrm{g} /$ jour...$\ldots \ldots \ldots \ldots \ldots$ & 6,7 & 7,15 & 7,0 & 7,25 & 6,7 & 7,15 & 7,0 & 7,25 \\
\hline Acide aspartique $\ldots \ldots \ldots \ldots \ldots$ & 0,4 & 0,5 & 0.4 & 0,4 & 3,1 & 5,2 & 4,1 & 6,0 \\
\hline Thréonine...$\ldots \ldots \ldots \ldots \ldots \ldots$ & 5,3 & 5,1 & 4,6 & 5,1 & 23,9 & 20,5 & 18,6 & 18,8 \\
\hline Sérine + glutamine $\ldots \ldots \ldots \ldots \ldots$ & 12,9 & 12,1 & 11,6 & 12,0 & 105.5 & 84,0 & 91,4 & 95,6 \\
\hline Acide glutamique..$\ldots \ldots \ldots \ldots$ & 2,6 & 2,5 & 2,7 & 4,0 & 45,6 & 42,4 & 41,3 & 39,8 \\
\hline 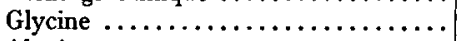 & 2,1 & $\overrightarrow{2,1}$ & 2,2 & 2,2 & 38,3 & 34,2 & 35,1 & 38,3 \\
\hline Alanine $\ldots \ldots \ldots \ldots \ldots \ldots \ldots \ldots$ & 4,1 & 3,6 & 3,6 & 3,4 & 32,9 & 29,1 & 30,3 & 29,2 \\
\hline 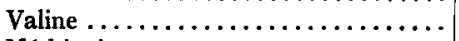 & 1,0 & 1,0 & 1,1 & 1,1 & 0,9 & 0,9 & 1,0 & 1,1 \\
\hline Méthionine ................... & 0,7 & 0,8 & 0,7 & 0,7 & 0,7 & 0,6 & 0,7 & 0,6 \\
\hline Isoleucine $\ldots \ldots \ldots \ldots \ldots \ldots \ldots$ & 0,4 & 0,5 & 0,5 & 0,6 & 0,3 & 0,3 & 0,5 & 0,6 \\
\hline Leucine $\ldots \ldots \ldots \ldots \ldots \ldots \ldots \ldots$ & 0,8 & 0,9 & 1,1 & 1,2 & 0,7 & 0,7 & 1,0 & 1,2 \\
\hline Tyrosine..$\ldots \ldots \ldots \ldots \ldots \ldots \ldots$ & 0,9 & 0,9 & 0,8 & 0,9 & 1,5 & 1,2 & 1,1 & 1,3 \\
\hline Phénylalanine.$\ldots \ldots \ldots \ldots \ldots \ldots$ & 0,8 & 0,8 & 0,9 & 0,9 & 0,9 & 0,7 & 0,9 & 0,7 \\
\hline Ornithine $\ldots \ldots \ldots \ldots \ldots \ldots \ldots \ldots$ & 0,5 & 0,5 & 0,5 & 0,6 & 1,5 & 1,2 & 1,3 & 1,2 \\
\hline Lysine $\ldots \ldots \ldots \ldots \ldots \ldots \ldots \ldots$ & 3,9 & 4,3 & 6,0 & 9,2 & 10,7 & 11,7 & 21,7 & 27,9 \\
\hline Histidine $\ldots \ldots \ldots \ldots \ldots \ldots \ldots \ldots$ & 0,9 & 0,8 & 1,0 & 1,3 & 3,8 & 3,8 & 3,7 & 3,8 \\
\hline Arginine $\quad \ldots \ldots \ldots \ldots \ldots \ldots \ldots \ldots$ & 1,8 & 1,8 & 2,0 & 2,5 & 3,4 & 3,5 & 3,6 & 4,8 \\
\hline
\end{tabular}

\section{TABLEAU 9}

\section{Expérience IV}

Teneurs en acides aminés libres (en $\mathrm{mg}$ pour roo $\mathrm{g}$ de tissu frais)

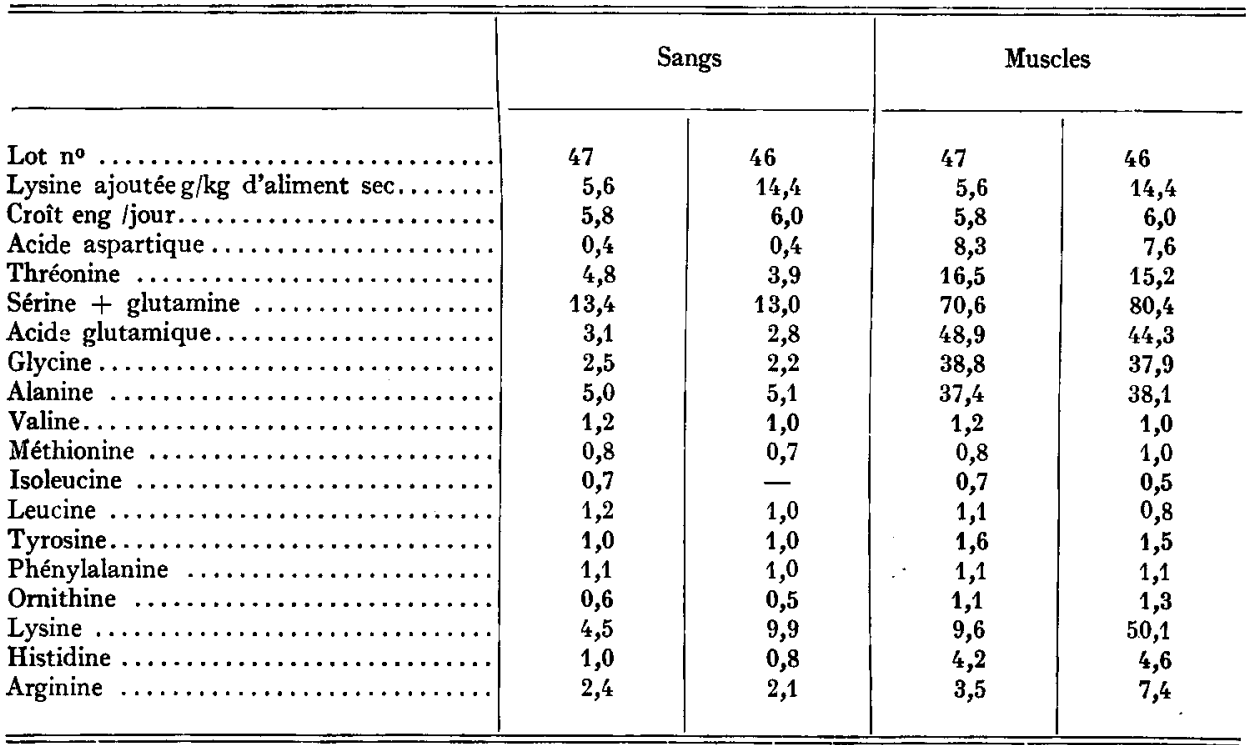


Infuence de la teneur en lysine de la ration.

Lysine (fig. I).

La teneur en lysine libre du sang augmente proportionnellement à celle de 1'aliment, lorsque cette dernière passe de 2,9 (blé non supplémenté) à $8,5 \mathrm{~g}$ par $\mathrm{kg}$ de matière sèche ingérée. Une augmentation supplémentaire de Io p. Ioo de la teneur en lysine de l'aliment entraîne une augmentation de $30 \mathrm{p}$. Ioo environ de la concentration de cet acide aminé dans le sang. La courbe de concentration semble présenter ensuite un plateau, redevient à nouveau ascendante lorsque la dose de lysine alimentaire passe de I I,7 à I2,5 g par kg d'aliment sec, puis atteint un nouveau plateau au-dessus de ce taux.

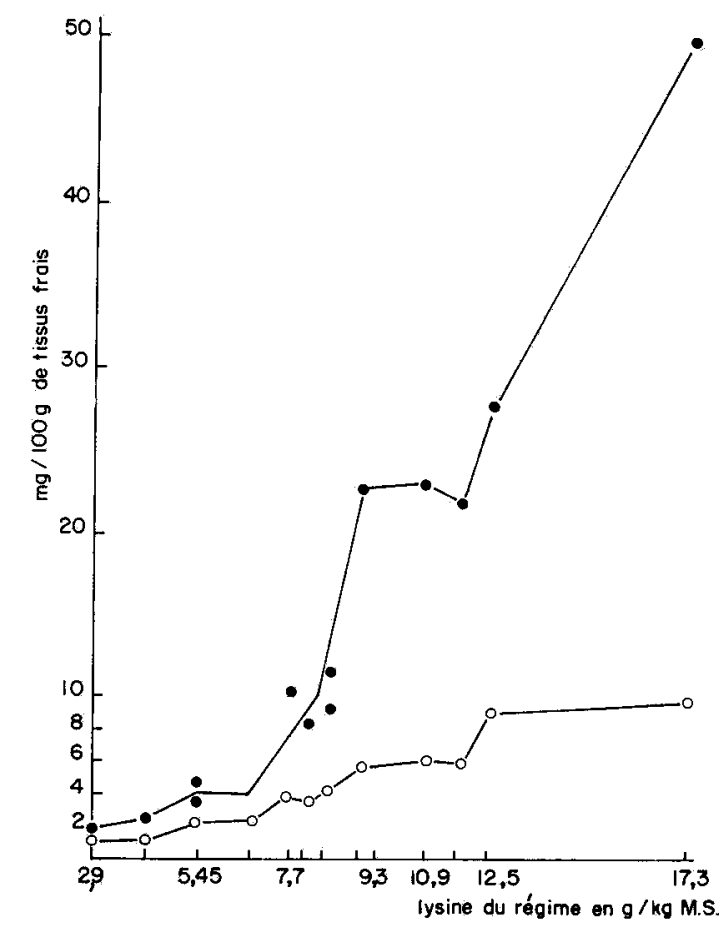

FIG. 1. - Teneurs en lysine libre des sangs et des muscles en fonction des doses croissantes de lysine du régime

- muscles 0 -o sangs

Les différences de concentration observées au niveau du tissu musculaire sont beaucoup plus nettes; les teneurs augmentent graduellement pour les plus faibles taux de supplémentation et sont 6 fois plus élevées chez les animaux ayant reçu une ration contenant $8,5 \mathrm{~g}$ de lysine par $\mathrm{kg}$ d'aliment sec que chez ceux qui ont ingéré le blé non supplémenté en lysine. Une addition supplémentaire de lysine $(9,3$ au lieu de $8,5 \mathrm{~g}$ par $\mathrm{kg}$ de matière sèche ingérée) entraîne une augmentation brutale de la teneur du muscle qui est doublée. Des quantités plus élevées comprises entre 9,3 et II,7 g par kg n'entraînent plus d'augmentation de la teneur en lysine libre du muscle. Cellesci se manifestent à nouveau pour des supplémentations plus importantes. 
Il y a donc un parallélisme entre les phénomènes observés au niveau du sang et au niveau du muscle, mais l'amplitude des variations est beaucoup plus importante dans le cas de ce dernier. Les concentrations en lysine libre du muscle et du sang des rats recevant la plus forte supplémentation sont respectivement 28 fois et 7 fois plus élevées que celles des animaux qui consomment le régime le plus pauvre en cet acide aminé, ce qui semble indiquer qu'il y a une accumulation importante de lysine dans le muscle.

\section{Thréonine.}

Elle a été apportée dans les rations en quantité légèrement supérieure à l'estimation du besoin du Rat (IIO p. IOo), de manière à ce que celui-ci soit certainement satisfait. Les teneurs en thréonine du sang et du muscle des animaux consommant les rations déficientes en lysine sont très élevées, et traduisent une certaine accumulation de cet acide aminé, dont 1'utilisation par les tissus est limitée par la faible quantité de lysine qui est mise à leur disposition. Elles tendent à diminuer avec la supplémentation en lysine des rations, en particulier dans le cas du muscle. Toutes les teneurs en thréonine observées aussi bien dans le sang que dans le muscle au cours de l'expérience I et IV sont inférieures à celles qui ont été mesurées pour les autres lots.

\section{Autres acides aminés.}

Il ne semble pas que l'influence de la teneur en lysine de la ration ait d'influence très nette sur les concentrations dans les tissus sanguins et musculaires des autres acides aminés indispensables; il en est de même dans la majorité des cas des acides aminés non indispensables.

\section{DISCUSSION}

\section{Méthodologie}

\section{Choix de la période expérimentale.}

La période choisie, dans la phase prépubertaire de la croissance, est caractérisée par une synthèse protéique intense, favorable à l'étude de l'utilisation des acides aminés, et à la mise en évidence des carences et des déséquilibres éventuels. Le poids des animaux au début de l'expérience est suffisant pour leur permettre de supporter sans trop de dommages des carences alimentaires relativement importantes, et de consommer la ration expérimentale en quantité suffisante pour que l'on puisse en mesurer effectivement les effets.

La durée de l'expérience est suffisamment longue pour éliminer l'influence du changement de régime, qui n'influe sur la consommation que pendant les trois premiers jours.

\section{Composition des régimes.}

Ils ont été établis de manière à assurer, dans l'état actuel de nos connaissances, la couverture des besoins des animaux sans excès notables, à l'exception du besoin en lysine, et leur ingestion a assuré des croissances élevées pour tous les lots dont les rations n'étaient pas trop fortement carencées en lysine. Les estimations des besoins en acides aminés utilisées sont celles de RANHOTRA et JoHNSON (I965) correspondant à des vitesses de croissance élevées. 
Prélèvements.

HILI, et OLSEN (I963), confirmant des travaux antérieurs, ont montré chez le Poulet qu'un jeûne de 12 'à 48 heures entraînait une modification importante des teneurs en acides aminés libres du plasma, et, en particulier, une accumulation de lysine et de thréonine. Aussi, dans cette expérience, les rats, qui avaient constamment de la nourriture à leur disposition, et n'étaient pas astreints à des repas, n'ont-ils pas subi de jeûne avant les prélèvements. Ils ont été sacrifiés entre to et I 2 heures du matin, après avoir pu s'alimenter normalement pendant la journée et la nuit précédente.

Conservation des échantillons et extractions.

Les dosages ont été effectués sur le sang total pour les raisons qui ont été exposées précédemment (PION, FAUCONNEAU et RÉRAT, I964). Les muscles prélevés ont été choisis en fonction de leur taille et de leur facilité de prélèvement qui permettent une fixation rapide des échantillons par congélation dans 1'azote liquide.

Les extraits alcooliques obtenus à partir des sangs se conservent très facilement à $-15^{\circ} \mathrm{C}$; les muscles congelés nécessitent plus de précautions : une décongélation accidentelle de courte durée modifie fortement leurs teneurs en acides aminés libres. Nous avons constaté qu'une décongélation d'une demi-heure pouvait multiplier les teneurs en acides aminés libres par 2 ou 3. Aussi, les échantillons ont-ils été maintenus congelés et à basse température jusqu'au moment où ils ont été broyés dans 1'acide picrique. Les teneurs en acides aminés de ces extraits picriques sont identiques, aux erreurs expérimentales près, pour la plupart des acides aminés, et, en particulier, pour tous les acides aminés indispensables dosés, à celles que 1'on peut observer dans les extraits alcooliques obtenus à partir des mêmes échantillons. La technique d'épuisements successifs présente une bonne sécurité, puisque près de $90 \mathrm{p}$. Ioo des acides aminés se trouvent dans le premier extrait.

\section{Résultats}

\section{Croissance.}

Les augmentations de croissance obtenues par l'addition au blé de doses croissantes de lysine n'ont été accompagnées que de faibles augmentations de consommation, et même, pour les taux les plus élevés utilisés dans l'expérience I, d'une baisse de consommation. La supplémentation se traduit donc par une amélioration de la transformation de l'aliment en tissus animaux, et $\mathfrak{n}$ 'intervient que faiblement sur la consommation. Ce fait, qui semble être en contradiction avec l'augmentation de la quantité d'aliment ingérée généralement associée à la supplémentation en lysine, est probablement dû̀ à ce que la consommation de ceux de nos animaux, dont la ration était la plus carencée, était déjà très élevée.

Des croissances de 5.7 à $6 \mathrm{~g}$ par jour ont été obtenues avec des rations ne contenant que $5,5 \mathrm{~g}$ de lysine par gramme de matière sèche ce qui laisse supposer que la lysine alimentaire est utilisée plus économiquement chez les animaux carencés, ou que les tissus synthétisés par ces animaux contiennent moins de lysine. Ce dernier fait pourrait être le corollaire de l'augmentation de l'incorporation de thréonine dans les protéines, constatée par YoshmDA et al. (I966), chez des animaux dont la ration comportait un large excès de thréonine.

Le fait que nous n'ayons pas constaté de diminution de la croissance chez les animaux dont les rations comportaient un excès de lysine n'est pas en contradiction 
avec les baisses notables observées par de nombreux auteurs, et en particulier par RÉRAT, BouFfaulT et JACQuot (I956), car les doses utilisées par ces derniers étaient beaucoup plus fortes que les nôtres. Il est, en revanche, en désaccord avec les observations de ZIMMERMan et Scotr (1965), qui ont observé dans des conditions voisines des nôtres une diminution de croissance de 20 p. Ioo chez le Poulet.

Les consommations alimentaires et les croissances observées au cours des expériences I et IV sont plus faibles que celles qui ont été obtenues pour des lots comparables au cours des expériences II et III. Ces différences sont peut-être dues à la nature du blé utilisé, qui avait été conservé un an à la température ambiante dans le cas des expériences I et IV.

Teneur en acides aminés libres du sang et $d u$ muscle.

Lysine.

Le changement de pente des courbes de concentration observé aussi bien dans le cas du sang que dans celui du muscle lorsque la teneur en lysine de l'aliment dépasse $8,5 \mathrm{~g}$ par $\mathrm{kg}$ de matière sèche, laisse supposer que le besoin en cet acide aminé est alors satisfait, qu'il y a un certain stockage des excédents dans les tissus, et, en particulier, dans le tissu musculaire. Il doit donc être possible d'utiliser ces courbes pour mesurer le degré de satisfaction du besoin en lysine des animaux à la suite de l'ingestion d'une ration donnée, et, par conséquent, le degré de disponibilité de la lysine des protéines qu'elle contient. La grande amplitude des variations observées au niveau du tissu musculaire permet d'espérer obtenir des résultats plus précis par l'étude de ce tissu que par celle du sang.

$\mathrm{L}_{\mathrm{a}}$ valeur obtenue pour le besoin est nettement plus faible que celle qui est généralement admise. Ce fait peut être dû aussi bien à la souche et aux conditions d'élevage des animaux utilisés qu'à l'équilibre de l'ensemble de la ration.

Nous n'avons pas observé d'accumulation importante de lysine dans le sang des animaux dont la ration contenait un large excès de cet acide aminé, contrairement à ce qu'ont observé McLaughidan et IlLMan (I967) chez le Rat et ZimMERman et ScoTr (1965) chez le Poulet, dans des conditions expérimentales assez différentes des nôtres. En revanche, la nette accumulation de lysine libre dans le tissu musculaire constatée chez les animaux ayant reçu une dose de lysine alimentaire sensiblement égale au double du besoin, permet de supposer que cet acide aminé n'est catabolisé que lentement par l'organisme, et qu'il peut être stocké au moins pendant un certain temps. Il est donc possible qu'une protéine alimentaire déficiente en lysine puisse être supplémentée efficacement par de la lysine libre provenant d'une ingestion antérieure ou d'une absorption plus rapide.

\section{Autres acides aminés.}

Les différents acides aminés indispensables sont présents en quantité relativement faible dans les prélèvements de sang et de muscle que nous avons analysés, à l'exception de la thréonine, ce qui confirme les résultats obtenus par G. WELLERS, J. CHEVAN et N. GalENT (1966). C'est peut-être pour cette raison que nous n'avons pas pu mettre en évidence pour les autres acides aminés la diminution de teneur observée dans le cas de la thréonine lorsque la teneur en lysine de la ration augmente.

Les teneurs en thréonine observées chez les animaux ayant consommé du blé stocké pendant un an sont plus faibles, aussi bien dans le cas du sang que dans celui du 
muscle, que chez les animaux des deux autres expériences. Il est donc possible que la faiblesse relative des croissances observées soit due, au moins en partie, à une indisponibilité partielle de la thréonine du blé.

\section{CONCLUSION}

Ces résultats montrent que la détermination de la teneur en lysine libre du tissu musculaire peut permettre une mesure précise de la satisfaction du besoin des animaux en cet acide aminé, à condition que la ration ne contienne pas d'autres facteurs limitants. Cette méthode peut être appliquée aussi bien à la détermination du besoin d'animaux ingérant une ration de composition bien connue qu'à la mesure in vivo de la disponibilité de la lysine des aliments. Il est vraisemblable, d'après les variations de teneurs observées dans le cas de la thréonine, qu'une méthode similaire peut être mise au point pour cet acide aminé. En outre, les croissances élevées observées dans la plupart des cas chez les animaux dont les rations n'étaient pas trop déficientes en lysine permettent de supposer que les acides aminés du blé, au moins lorsqu'il n'a pas șubi de longue conservation, sont bien utilisés par l'animal.

Reçu pour publication en juin 1968.

\section{REMERCIEMENTS}

Ce travail a été réalisé en partie grâce au contrat de la Délégation générale à la Recherche scientifique et technique $n^{\circ} 67008 \mathrm{rg}$.

\section{SUMMARY}

INFLUENCE OF SUPPLEMENTING WHEAT PROTEINS WITH INCREASING AMOUNTS OF LYSINE ON THE FREE AMINO ACID CONTENT OF BLOOD AND MUSCLE OF GROWING RATS

The study was made on Wistar rats reared in individual cages and fed on diets based on wheat supplemented with amino acids (tables 4 and 5 ).

The requirements of amino acids except for lysine were covered by all diets. The different diets had graded amounts of lysine between 30 and 200 per cent of the theoretical requirement of the animals (table 5 ). At the end of the exporimental period ( 2 weeks) whole blood and muscle of the hind limbs were sampled. After extraction with cold $84 \mathrm{p}$. 100 ethanol for blood and Io $\mathrm{p}$. I00 picric acid for inuscular tissue, free amino acids were estimated by column chromatography.

Growth rates obtained were high except for diets with the lowest contents of lysine. Intake of food was always high and differed little as a result of supplementary lysine.

Free lysine in blood and muscle increased with that in the feed (fig. I), but the degree of difference was much higher in the muscular tissue. The free lysine content of muscle in rats given the diet richest in lysine was 27 times greater than that in animals eating wheat not supplemented with lysine, while the highest value seen in blood was only 7 times the lowest. In both tissues there 
was a large increase when the requirement of the animal was satisfied. The contents of other amino acids changed little, except for threonine which accumulated in blood, and particularly in muscle in the case of a deficit of lysine.

The particularly large increase of lysine seen in muscle when the requirement of lysine was met shows that measurement of this concentration could be a particularly sensitive method for estimation of the degree to which requirement of this amino acid is satisfied.

\section{RÉFÉRENCES BIBLIOGRAPHIQUES}

Harper A. E., Rogers Q. R., 1965. Amino acid imbalance. Proc. Nutr. Soc., 24, i 73-190.

HILL D. C., OLSEN E M., 1963. Effect of starvation and a non protein diet on blood plasma amino acids and observations on the detection of amino acids limiting growth of chicks fed purified diets. $\quad J$. Nutr., 79, 303-310.

LONGENECKER J. B., HAUSE N. L., I I59. Relationship between plasma amino acids and composition of the ingested proteins. Archs. Biochem. Biophys., 84, 46-59.

McLaughlan J. M., Illman W. I., 1967. Use of free plasma amino acids levels for estimating amino acid requirements of the growing rats. J. Nutr., 93, $2 \mathrm{I}-24$.

Moore S., Spackman D. H., Stein W. H., 1958. Chromatography of amino acids on sulfonated polystyrene resins. Analyt. Chem., 30, 1185 - I 196.

Morrison A. B., Middeteton E. J., McLaughi.an J. M., 196r. Blood amino acid studies. II. Effect of dietary lysine concentration, sex, and growth rate on plasma free lysine and threonine levels in the rat. Can. J. Biochem. Physiol., 19, I 676-1680.

PAWLAK M., PION R., I 967 . Influence de la composition des protéines alimentaires sur les teneurs en acides aminés libres du sang total et du muscle du rat en croissance. C.R. H. Acad. Sci., Paris, 264, 380-382.

PION R., Fauconneau G., Rérat A., I964. Variation de la composition en acides aminés du sang porte au cours de la digestion chez le porc. Annl. Biol. anim. Biochim. Biophys., 4, 383-40I.

Rérat A., Bouffault J. C., JACQUOT R., 1956. Double limitation de l'efficacité protidique par carence ou par excès du même acide aminé indispensable démontrée par l'aptitude de la DL-lysine à suppléer le gluten de mais ou de blé. C. R. Acad. Sci., Paris, 243, I 92-1 94.

Spackman D. H., Stein W. H., MoORE S., r958. Automatic recording apparatus for use in the chromatography of amino acids. Analyt. Chem., 30, I $190-1206$.

TAllan H. H., Moore S., Stein W. H., I954. Studies on the free amino acids and related compounds in the cat. J. Biol. Chem., 211, 927-939.

Wellers G., Chevan J., Galent N., 1966. Les pools d'acides aminés libres dans les tissus du rat normal. C. R. Soc. Biol., 160, 739-742.

Yosh IDA A., LeUngP M. B., Rogers Q. R., HARPER A. E., 1966 . Effect of amino acid imbalance on the fate of the limiting amino acid. J. Nutr., 88, 80-90.

Zimmerman R. A., ScotT H. M., r 965 . Interrelationship of plasma amino acid levels and weight gain in the chick as influenced by suboptimal and superoptimal dietary concentration of single amino acid. J. Nutr., 87, $13-18$. 